\title{
ROAD VEHICLES IDENTIFICATION AND POSITIONING SYSTEM
}

\author{
Cemil SUNGUR \\ Selcuk University, School of \\ Technical Sciences.42250 Campus- \\ KONYA \\ Email: csungur@selcuk.edu.tr
}

\author{
Hacı Bekir GÖKGÜNDÜZ \\ Selcuk University, School of \\ Technical Sciences.42250 Campus- \\ KONYA \\ Email: gokgunduz@selcuk.edu.tr
}

\author{
Adem Alpaslan ALTUN \\ Selcuk University, Faculty of \\ Technology.42250 Campus - \\ KONYA \\ Email: altun@selcuk.edu.tr
}

\begin{abstract}
Radio frequency identification (RFID) is untouched automatic identification technologies in which information can be transmitted by radio frequency. In this study, an RFID system which can determine the position and place of any desired vehicle is designed and developed. Microprocessor transmitter devices, on which the vehicle information is loaded, are placed on the vehicles. Receiver circuits are located at certain points in order to receive the RF signals sent by these transmitter devices. The communication of all the points with each other is maintained by connecting the receiver circuits via the internet. The functionality of the system is tested by performing receiver-transmitter experiments based on the various speeds and locations of the vehicles. According to the results obtained in the experiments, it is seen that the system designed in the study could be used in place of the GPS system for determining the place and position of vehicles, since it is more economical when compared to the GPS system.
\end{abstract}

\section{INTRODUCTION}

$\mathrm{N}$ owadays, private organizations, individuals, and particularly public institutions have a rapidly increasing need for the positional information of various objects. The increase in the demand for information and in variety requires the collection of the positional information of individuals, institutions and regions in a faster and more economical way. The desire to maintain continuity and speed in the currency of information has resulted in rapid developments in Global Positioning Systems (GPS). The need for information can be evaluated on three basic scales, as local, regional and global.

The use of GPS systems is expensive and limited, and such systems have strategic importance. For this reason, it is necessary to create alternative systems. Different from the systems that are established and used by developed countries and the use of which by other countries is restricted, in the present study, a system whose use is not dependent on the permission of other countries, which does not require the use of satellites and which is considerably economical when compared to other systems is designed. Vehicle recognition, tracking and positioning system is implemented in order to determine the location and position of vehicles by using RF

This work was not supported by any organization signals. In this way, although not point-wise, it is possible to determine the position of a vehicle in a narrow area.

Various results have been obtained in previously conducted similar studies [1]. A vehicle management system based on UHF band RFID technology is proposed in [2]. This system is applied to vehicles entering/leaving at road gates. The system consists of tag-on-car, reader antenna, reader controller, and monitoring and commanding software. Schneider proposes radio frequency identification technology for potential applications in the commercial construction industry [3]. It is defined that RFID can increase the service and performance of the construction industry with applications in materials management, tracking of tools and equipment, automated equipment control, jobsite security, maintenance and service, document control, failure prevention, quality control, field operations, and construction safety. Tu et al. examine radio frequency identification (RFID) tags, which are increasingly being used in pervasive healthcare applications [4]. Specifically, they study the dynamics of locating and identifying the presence of a tag in such systems. In [5], web servers build the agents by themselves and an agent-based interaction works with the support of Web services. Thus, they supposed to build an agent-based structure for transportation control that is similar to the structure of the Internet. Agent-based transportation management is a possible contribution to make transportation management more effective in regard to saving energy (fuel) and protecting our environment by stopping the increase of existing traffic ways. Liu et all. study on a platform for moving vehicle detection and tracking [6]. A novel Marr wavelet, kernel-based background modeling method and a background subtraction method based on binary discrete wavelet transforms (BDWT) are introduced. In addition, an automatic particle filtering algorithm is used to track the vehicle after detection and obtaining the center of the object. Böse et al. investigate an innovative approach to autonomous control in automobile logistics, considering as example the logistic order processing of an idealized automobile terminal of the company [7]. They present the results of an executed case study concerning the implementation and test of an 
autonomously controlled, radio frequency identification (RFID) based storage management system. In [8], an invehicle signing system is built and assessed that uses general-purpose RFID tags as digital traffic signs, and a field test is conducted using tags installed on a road to verify whether the system works effectively. In [9], a radio frequency identification (RFID) system for defining railway vehicles and an antenna is designed for the receiver/transmitter circuit of the RFID system is proposed. An intelligent traffic management system by using RFID technology is proposed in [10]. Using the system, important traffic and control data is obtained in a practical way, and also illegal vehicles, such as those which have been stolen are tracked.

\section{GLOBAL POSITIONING SYSTEM}

Global Positioning System (GPS) is a satellite network which conveys coded information in a regular manner. This system is composed of 24 satellites which continuously move in orbit and broadcast very low-power radio signals. The GPS receiver on the Earth's surface makes it possible to determine our definite position on the Earth by receiving those radio signals and measuring our distance from the satellites [11]. The functioning structure of the global positioning system is presented in Figure 1.

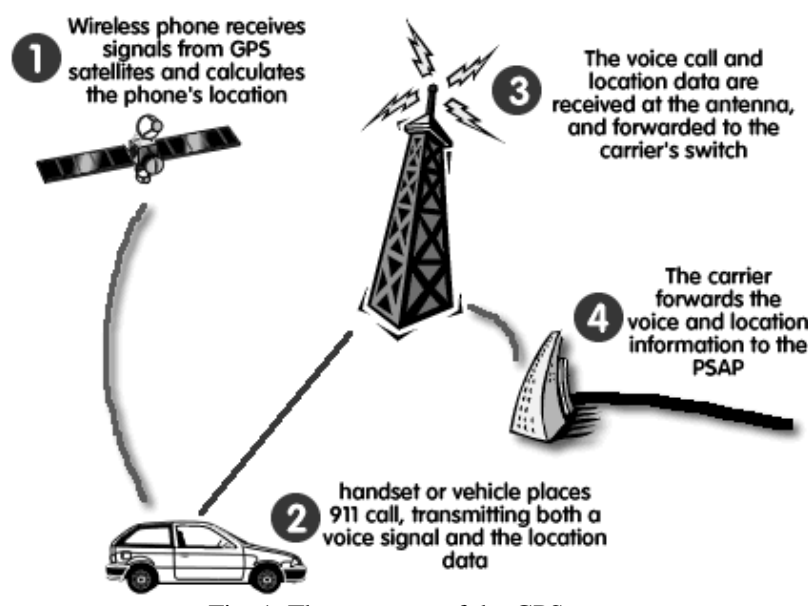

Fig. 1. The structure of the GPS system

The structure of the GPS system is composed of three major parts: the space segment, the control segment and the user segment. The space segment consists of at least 21 active satellites and 3 on-orbit spares. Each of the satellites transmits radio signals on two different frequencies. Civilian GPS satellite signals are transmitted at a frequency of 1575.42 MHz in the UHF band, while military GPS signals are transmitted at a frequency of $1227.6 \mathrm{MHz}$ and are used by the US defense unit. Each satellite broadcasts two special pseudo-random codes which enable the receivers on the Earth to recognize the signals. These signals include the orbit, time, general system and ionospheric delay information of the satellite. The control segment obtains the exact orbit and time information by continuously tracking the satellites. There are several control stations on the surface of the Earth [12]. The user segment is comprised of all of the GPS receivers on the Earth.

The GPS tracking system was originally intended for military applications, but in the 1980s, the system was made available for civilian use upon the increasing demand. The proportion of the civilian use of GPS systems has reached a high rate of $90 \%$ compared to its use for military purposes [13].

\section{RF COMMUNICATION}

Communication technology, which has gained a new dimension and speed in recent years, has led to the development of techniques that enable the use of the air as a transmission environment and the sending of information to the desired point by coding the signal on a high frequency carrier wave, which is also named as modulation.

An electromagnetic wave is formed when an electric field combines with a magnetic field. These waves, which are combined and sent by the RF transmitter, are separated by the $\mathrm{RF}$ receiver and transformed into electric and magnetic waves [14].

Devices which are used for modulating the amplitude, frequency or the phase of the information signal and the high frequency carrier signal and then transmitting the information through the air or a conductive environment are called RF transmitters. RF receivers are devices by which the signals coming from the RF transmitter are received by an antenna and amplified by the power amplifier, then mixed with the local oscillator signal. Then, the RF signal is transformed to information by being demodulated at the output of the mixer. The operating principles of RF transmitter and receiver circuits are presented in Figure 2 [14].

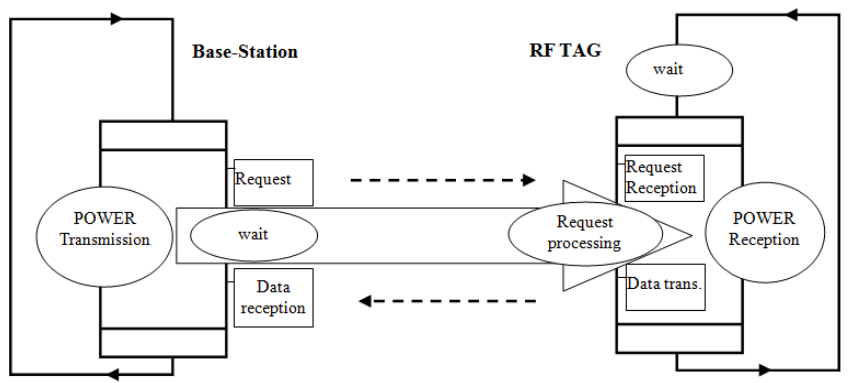

Fig. 2. RF Receiver and Transmitter Circuit Block Diagram

As shown in Figure 2, RFID system is composed of tags, which carry the data in suitable transponders, and an RFID reader, which retrieves the data from the tags, and software such as a driver and middleware. The main function of the RFID system is to retrieve information (ID) from a tag (also known as a transponder). Tags are usually affixed to objects such as goods or vehicles so that it becomes possible to locate where the goods and vehicles are without line-of- 
sight. A tag can include additional information other than the ID, which opens up opportunities to new application areas. In these systems, the RF area is broken down into a number of grouped frequencies that are called bands. The frequencies used in RFID systems are analyzed below:

- Less than $135 \mathrm{kHz}$ : This is the low frequency (LF) which allows the detection of RFID tags in a short range. The data reading speed is low and in this frequency the electromagnetic waves penetrate water but not metal. This frequency is used for animal identification, inventory control and car immobilizer [15].

- 13.56MHz: This frequency, which is called as high frequency (HF), allows the detection of RFID tags for a distance of up to $1.5 \mathrm{~m}$. The data transfer rate for this specific frequency is approximately 25 Kbits per second. In this frequency, the electromagnetic waves can penetrate water but have poor performance around metal. This frequency is used for applications related to access, security payment and various item levels tracking such as books, luggage, etc. [16].

- 433MHz, 850-956MHz: The frequencies which belong to this range are characterized as ultra-high frequencies (UHF). This frequency allows high reading speed as approximately $100 \mathrm{Kbits}$ per second. The frequencies at this range are used for applications in vehicle tracking, railway vehicle monitoring [16].

- 2.45-5.8GHz: This frequency enables an RFID reader to detect a tag from a distance of ten meters. This specific frequency is characterized as microwave frequency. Data transfer rates are fast but the reading range is similar to UHF. The specified frequency is used for applications related to industrial automation [16].

In our vehicle tracking system, an ATX-34 circuit is used as the RF transmitter and an ARX-34 circuit is used as the RF receiver. ATX-34 and ARX-34 circuits are preferred for their high stability and wide area of use. These circuits comply with the radio standard EN 300220 at $433.920 \mathrm{MHz}$ UHF band, have high frequency stability and are ideal for battery applications with their low current consumption [17].

In this study, the PIC16F877 microcontroller manufactured by Microchip Technology Inc is used for entering the license plate information of the vehicles, and programming and storing the information coming from the transmitter [18]. This microcontroller is programmed using the JAL programming language [19]. The antenna used in the study has an impedance of $50 \Omega$ and is approximately $17.3 \mathrm{~cm}$.

The transmitter circuit is programmed together with the license plate information. The PIC16F877 integrated circuit can be locked against rewriting and reading during the programming process. In this way, the access of unauthorized persons to the license plate information of vehicles can be restricted. The license plate information is not transferred by using a standard on the transmitter circuit so that it cannot be easily acquired. The circuit transmits the license plate information through a completely specific protocol. Figure 3 shows the fundamental steps of the RFID system.

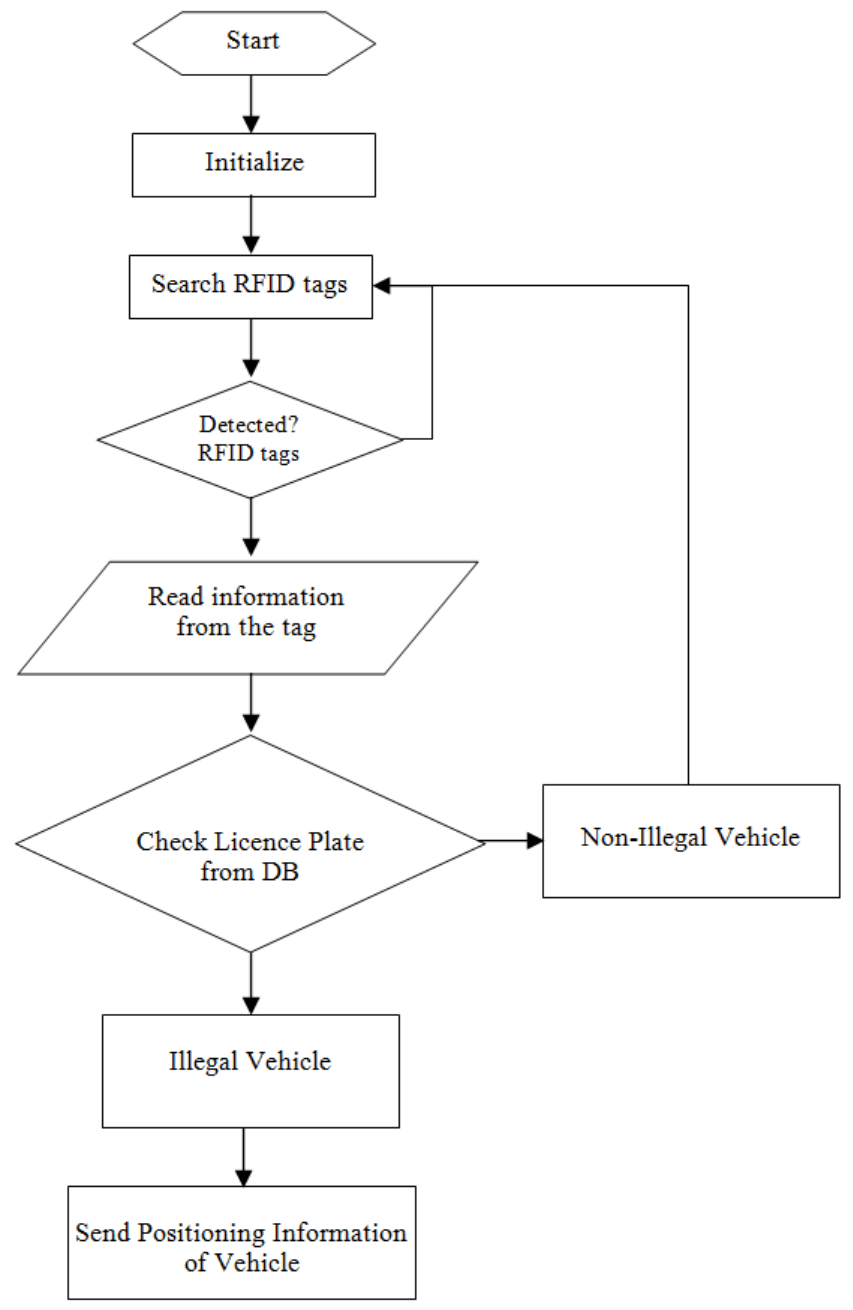

Fig. 3. Diagram of the RFID system for vehicle identification

In each transmission, the transmitter circuit sends a data package. The eight-character license plate information is transmitted in this data package as shown in Figure 4. The license plate information is added to the beginning of the preamble data package. The radio preamble is a section of data at the head of a packet that contains information the access point and the client devices need when sending and receiving packets. The preamble data is a leading signal which is composed of 6 bits and each period of which is $1 \mathrm{~ms}$. This signal is used in order to prevent the faulty reception of the initial data by a receiver. The faulty reception of the initial data is prevented by sending a leading preamble data. After this, the synchronous signal and the 8byte data are transmitted. The permanent duty of the transmitter circuit is to continuously broadcast the recorded license plate information.

\begin{tabular}{|l|l|l|l|l|l|l|l|}
\hline 4 & 2 & $\mathrm{~B}$ & $\mathrm{~V}$ & 0 & 2 & 1 & 3 \\
\hline
\end{tabular}


Fig. 4. 8-bit license plate information data

The general structure and functioning of the system is presented in the block diagram of the circuit shown in Figure 5.

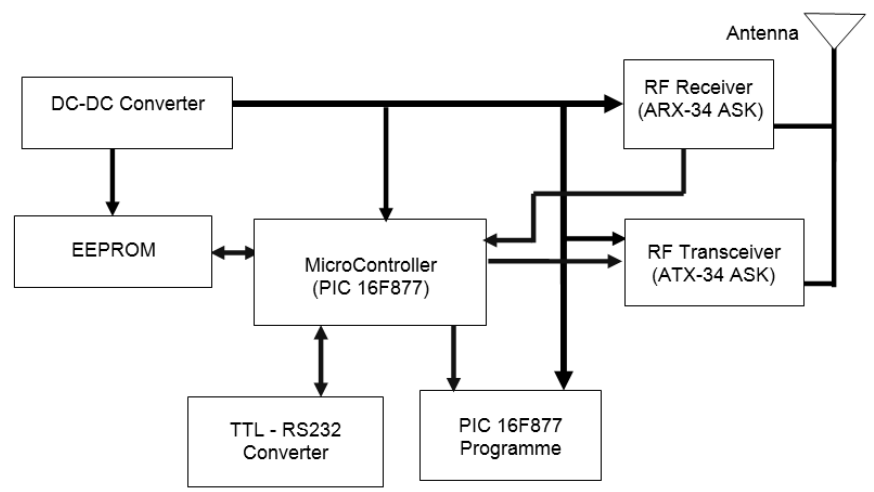

Fig. 5. The circuit block diagram of the designed RFID system

When the receiver circuit receives RF information from the transmitter circuit that complies with its protocol, it transmits this information to the computer through the RS232 port. The data is stored on the computer and the desired information can be selected. The searched license plates are reported to the program and the program searches for the wanted plates among the received data. When one of the searched license plates enters into the coverage area of the tracking system, the program records the entry with date and time, and displays an alert on the screen to warn the user. The circuit transmits the data which is only in a compatible format at a speed of $115.200 \mathrm{bps}$.

The signals received from the receivers are processed by the interface program. There is a transmitter circuit which has a specific code on each vehicle. As it can be seen in Figure 6, the interface consists of three main parts. The first of these is the Passing Vehicles Part, the second is the part where we enter the license plate information of the vehicle we want to find, and the third is the part where we determine at what time a certain vehicle passes a certain point.

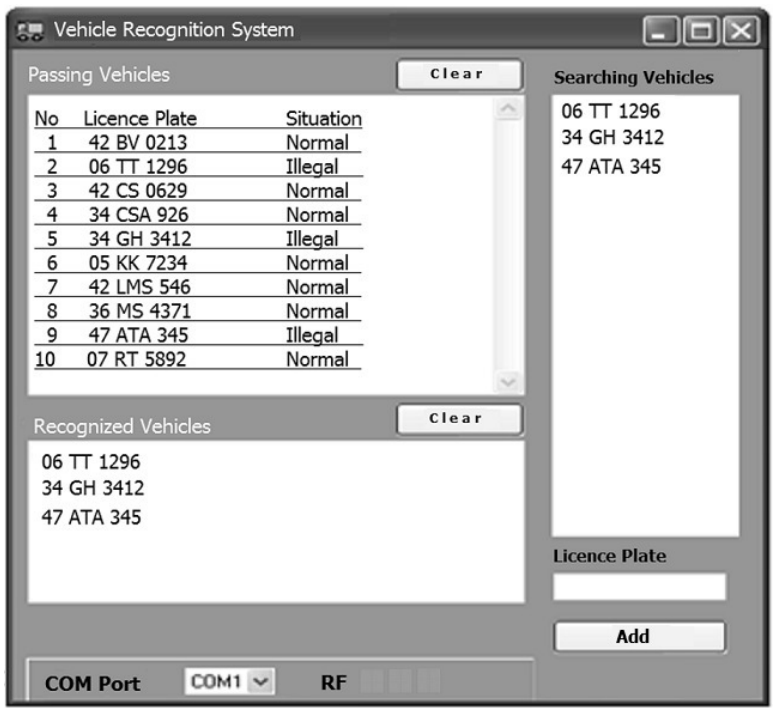

Fig. 6. The interface program developed for the vehicle tracking system

When the program is started, the information obtained in the three parts begins to be stored in the database. In this way, access is provided to the information concerning a desired time, day and hour.

\section{EXPERIMENTAL RESULTS}

Two transmitter and one receiver circuits are used in the system designed for this study. The receiver circuit is located at a point on the route determined for the study. The two transmitters are placed on two different vehicles. The vehicle information of each vehicle passing along the route is read by the RF receiver. In this way, it is known which city the vehicle travels to, where it stops over and from which direction the vehicle enters the city. An area on which the license plate information of the searched vehicles can be entered is added to the computer software used for recording and monitoring vehicle information. In this way, when the sought vehicle enters the coverage area of the receiver, the vehicle information is delivered to the system user through the computer.

The license plate information of the sought vehicle is entered to the system by the traffic data processing unit of the city and it can be seen by the traffic information centers of all the cities with the help of a computer network. When the vehicle is detected within the borders of a city, the location of the vehicle is transmitted to the system users of all the cities via the computer network.

In this study, it is planned to place the system at the entrance and the exit of the city. However, the system can also be used at traffic points, intercity traffic stations, intersections and highway entrance and exit booths.

In the present study, a total of five experiments are conducted on; the distance required for the recognition of the vehicles equipped with a transmitter by the receiver, the speed of the vehicle which is effective on recognition, 
weather conditions, the effect of the position of the receiver and the transmitter on the recognition of the vehicle.

\section{A.DETERMINATION OF THE RECOGNITION DISTANCE}

The receiver is placed $1 \mathrm{~m}$ above the ground, and the approach and deviation distances are found to be $30 \mathrm{~m}$ at the 10 passes made at a speed of $50 \mathrm{Km} / \mathrm{h}$ by the transmitter placed on the vehicle, as shown in Figure 7.

When the receiver is placed at a height of $2.20 \mathrm{~m}$, it is observed that the approach and deviation distances are found to be still equal at the 10 passes made at a speed of $50 \mathrm{Km} / \mathrm{h}$, but the recognition distance of the vehicle has increased to $50 \mathrm{~m}$.

When the receiver is placed at a height of $4.60 \mathrm{~m}$, it is observed that the approach and deviation distances are found to be still equal at the 10 passes made at a speed of $50 \mathrm{Km} / \mathrm{h}$, but the recognition distance of the vehicle has increased to $75 \mathrm{~m}$.

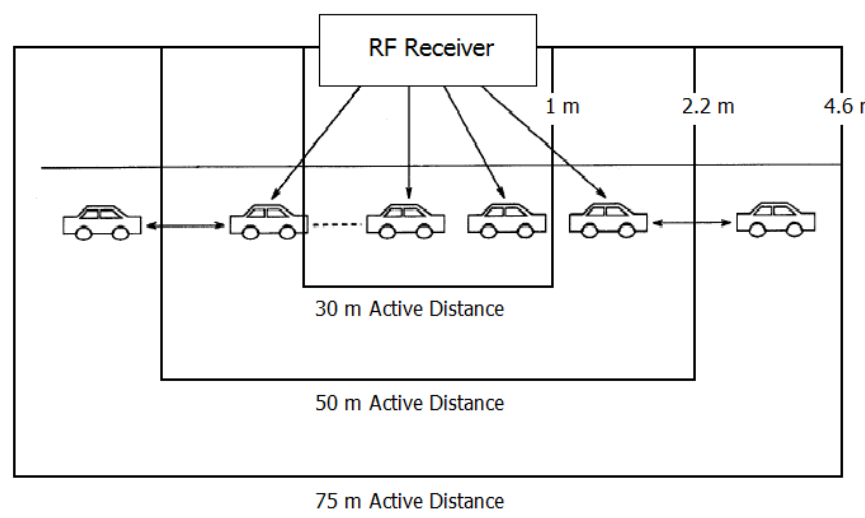

Fig. 7. The distances at which the transmitter is detected by the receiver

\section{B. DETERMINATION OF THE SPEED THAT PREVENTS THE RECOGNITION OF THE VEHICLE}

It is necessary that the vehicles passing the areas where the receivers are located be sensed by the receiver. The receiver may not sense the vehicle due to several factors, such as the passing of more than one vehicle simultaneously and the speed of the passing vehicle. The height of the receivers and the speed of the vehicles are important for sensing the passing vehicles in a reliable manner. For this reason, the receiver is placed at a height of $2.20 \mathrm{~m}$ and the approach and deviation distances of the vehicle are found to be the same at the passes made at speeds of $20 \mathrm{Km} / \mathrm{h}, 50$ $\mathrm{Km} / \mathrm{h}, 80 \mathrm{Km} / \mathrm{h}, 100 \mathrm{Km} / \mathrm{h}$ and $120 \mathrm{Km} / \mathrm{h}$, and the recognition distance of the transmitter by the receiver is found to be $50 \mathrm{~m}$.

\section{RECOGNITION NUMBER OF THE VEHICLE}

It is necessary that the receiver senses the vehicles passing the active region and obtains reliable data. For this reason, it is important how many times the receiver communicates with the transmitter in the active region. In this study, it is determined how many times the receiver recognizes the transmitter in the active region depending on the speed of the vehicle and the obtained number is compared with the calculations performed based on the frequency of the transmitter. When the period required for the wavelength of the transmitter broadcasting at a frequency of $433.927 \mathrm{MHz}$ is calculated.

$$
\mathrm{T}=1 / \mathrm{f}
$$

where; $\mathrm{T}$ is the period (s), $\mathrm{f}$ is the frequency $(\mathrm{Hz})$. Thus period is obtained as $2.3 \mathrm{~ns}$.

When the distance covered by a vehicle travelling at a speed of $100 \mathrm{Km} / \mathrm{h}$ is calculated.

$$
\mathrm{S}=\mathrm{V} / \mathrm{t}
$$

where; $\mathrm{S}$ is the distance traveled in $1 \mathrm{~s} ; \mathrm{V}$ is the speed of the vehicle $(\mathrm{m} / \mathrm{s})$; $\mathrm{t}$ is the total time $(\mathrm{s})$.

In Eq. 2, S is calculated as $100.000 / 3.600=27.7 \mathrm{~m}$. Therefore, the vehicle would pass the $50 \mathrm{~m}$ active region in 2 $\mathrm{s}$ considering the length of the vehicle (supposing that the vehicle is a passenger car).

A number of $2 \mathrm{~s} / 2.3 \mathrm{~ns}=0,869 \times 109$ signals are sent to the receiver from the transmitter. The vehicle license plate consists of 8 characters. Each character requires successive continuous data transfer at periods of $1 \mathrm{~ms}$. For this reason, information of a vehicle is sent over to the receiver every 8 ms. Moreover, when we add the starting and ending bits, this period is extended to $10 \mathrm{~ms}$. That is, a vehicle information reaches the receiver every $10 \mathrm{~ms}$, therefore the receiver can read the information from the transmitter $2 \mathrm{~s} / 10 \mathrm{~ms}=200$ times within a passing period of $2 \mathrm{~s}$. Each piece of information is read 4 times consecutively in the interface program to ensure that the vehicle is completely recognized. Thus, the vehicle information is read $200 / 4=50$ times. It is expected that this number of readings will be adequate for the receiver to read the transmitter without missing the receiver even if there is more than one vehicle in the active reading area.

The receiver is placed at a height of $2.20 \mathrm{~m}$ and the experiment is repeated by passing the transmitter by the receiver point 5 times at each speed of $20 \mathrm{Km} / \mathrm{h}, 50 \mathrm{Km} / \mathrm{h}$, $80 \mathrm{Km} / \mathrm{h}, 100 \mathrm{Km} / \mathrm{h}$ and $120 \mathrm{Km} / \mathrm{h}$.

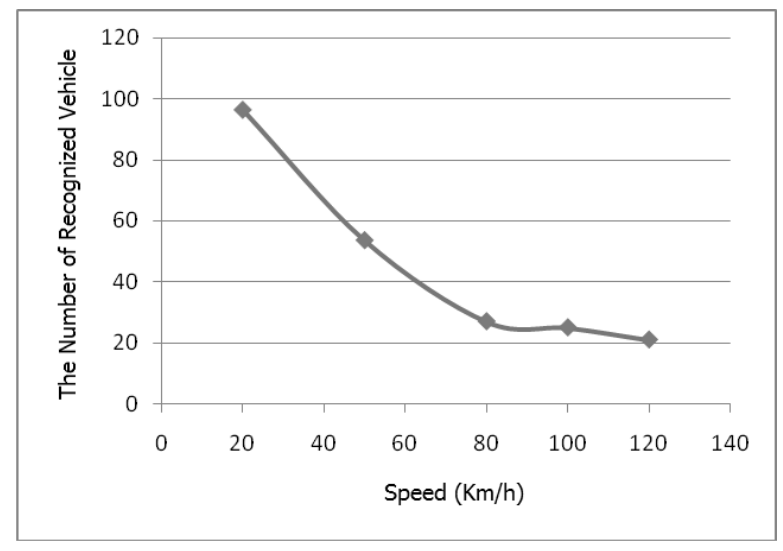

Fig. 8. Speed - Number of Detection Graph 
The data obtained as the result of this experiment is shown in Figure 8. As it can be seen in the graph, as the speed of the vehicles increase their number of detection decreases. The maximum speed limit is $120 \mathrm{Km} / \mathrm{h}$ in our country. For this reason, our experiment is conducted up to this limit.

When more than one vehicle enters the recognition distance of the receiver at the same time, the number of detection of each vehicle is measured separately. As it can be seen in Figures 9 and 10, the entry of two vehicles of the same type into the active area does not affect the detection of the vehicles. However, although the distance of detection remains the same, the number of detection decreases to half, even to $40-45 \%$ when compared to the experiment conducted with one vehicle.

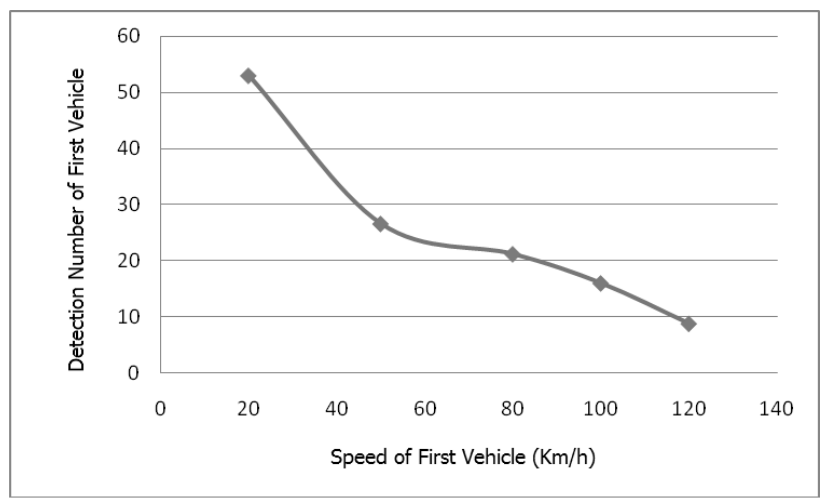

Fig. 9. 1st Vehicle Speed - Number of Detection Graph

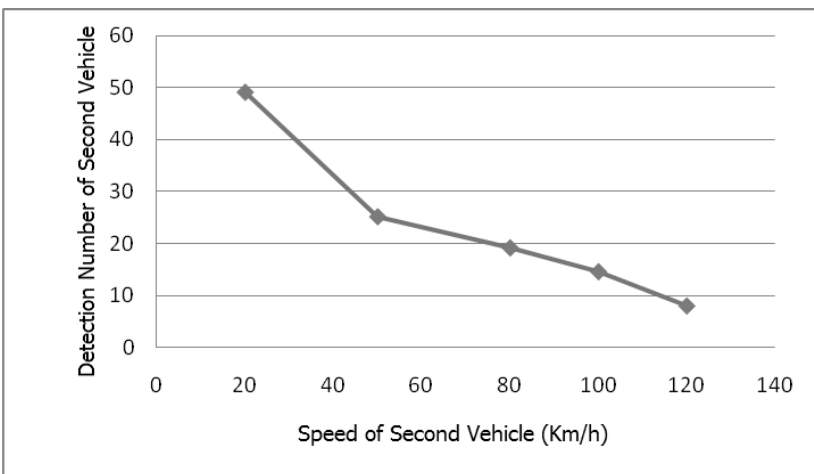

Fig. 10. 2nd Vehicle Speed - Number of Detection Graph

In a further experiment, the transmitter vehicle is passed by a heavy vehicle at different speeds as shown in Figure 11, and the values presented in Table 1 are obtained as the result of the experiment.

As it can be seen in Table 1, the number of detection of the transmitter vehicle which is behind the heavy vehicle significantly decreased. This could be considered as a disadvantage of our vehicle tracking system.

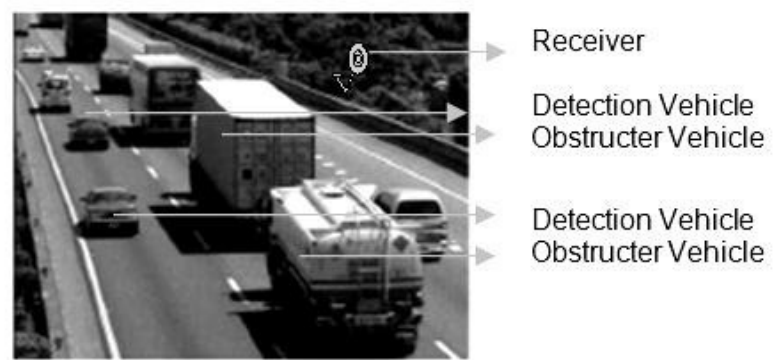

Fig. 11. Moment of passing of the undetected vehicles

TABLE I.

Number of Detection of a Vehicle behind Heavy Vehicles

\begin{tabular}{|c|c|c|c|c|c|c|}
\hline $\begin{array}{c}\text { Speed } \\
\mathbf{K m} / \mathbf{h})\end{array}$ & \multicolumn{5}{|c|}{ Number of Detection } & \multirow{2}{*}{ Mean } \\
\hline $\mathbf{2 0}$ & $\mathbf{1}^{\text {st }}$ Pass & $\mathbf{2}^{\text {nd }}$ Pass & $\mathbf{3}^{\text {rd }}$ Pass & $\mathbf{4}^{\text {th }}$ Pass & $\mathbf{5}^{\text {th }}$ Pass & \\
\hline $\mathbf{5 0}$ & 6 & 15 & 14 & 14 & 15 & 20.6 \\
\hline $\mathbf{8 0}$ & 3 & 2 & 8 & 7 & 8 & 6.8 \\
\hline $\mathbf{1 0 0}$ & 2 & 1 & 0 & 0 & 2 & 1.4 \\
\hline $\mathbf{1 2 0}$ & 0 & 0 & 0 & 0 & 1 & 0.8 \\
\hline
\end{tabular}

In this study, an RFID system for tracking and determining the position of vehicles is designed. In this system, all the vehicles on which transmitters are placed are detected and recognized by the system when they pass by the points where receivers are located. Experiments are conducted in order to determine the speed that prevents the recognition of the vehicle, to recognize each vehicle when more than one vehicle enters the recognition distance of the receiver at the same time and to determine the physical obstacles that might prevent the recognition of the vehicle. In the designed system, the license plate information of the vehicles can be viewed in the interface program. This information is recorded on the computer via the interface program. When the license plate information of the sought vehicle is entered into the interface program, the exact time when the vehicle passes a previously determined point is displayed as day, hour, minute and second. Furthermore, all the vehicles detected by the receiver are listed in the interface program. The process of determining the place and position of a vehicle, which can be carried out using the GPS system whose use is restricted, is easily performed. In this way, a moving vehicle can be tracked by using the system developed in this study.

It is observed that the performance of the system decreases when the experiments described above are performed under maximum conditions, that is, under very heavy traffic and unfavorable weather conditions. Under such conditions, the performance of the system can be enhanced by placing the receiver in a higher position, adjusting its direction and increasing the signal level of the transmitter.

The system designed in the present study can be adjusted in a manner that enables access to insurance, vehicle inspection, vehicle license and driving license information 
through certain changes and additional software. Access to the mentioned information can be rendered exclusive to certain institutions, organizations and individuals. In this way, it can be possible to receive information regarding when and from where a vehicle passes and also to access vehicle license and driving license information.

\section{Conclusion}

This research has investigated the practical issues of deploying the RFID technology for the vehicle identification started from hardware procurement process. The paper has researched several technical aspects of RF-ID implementation. Microprocessor transmitter devices, on which the vehicle information is loaded, are placed on the vehicles. Receiver circuits are located at certain points in order to receive the RF signals sent by these transmitter devices. The communication of all the points with each other is maintained by connecting the receiver circuits via the internet. According to the results obtained in the experiments, it is seen that the system designed in the study could be used in place of the GPS system for determining the place and position of vehicles, since it is more economical when compared to the GPS system.

The results of the experiments indicate that even implementing the system in a small scale (prototype system), there are a number of challenges to be solved in the middleware development phase, and extraordinary challenges in the configuration and tuning phases. Therefore to gain a cost effective system from deploying RFID for vehicle tracking in practice, extra attention is definitely required, and further experimentation on a real-life scale (e.g., real vehicle and gate) and/or using different sets of equipment will help confirm the results from this research.

\section{REFERENCES}

[1] Young CP, Chang BR, Tsai HF, Fang RY. and Lin JJ (2009) Vehicle Collision Avoidance System using Embedded Hybrid Intelligent Prediction based on Vision/Gps Sensing, International Journal of Innovative Computing, Information and Control, vol. 5 (12(A)), pp. 44534468

[2] Ning Y., Zhong-qin W., Malekian R., Ru-chuan W. and Abdullah A.H. (2013) Design of Accurate Vehicle Location System Using RFID, Elektronika Ir Elektrotechnika, Vol. 19, No. 8, pp. 105-110.
[3] Schneider M(2003) Radio Frequency Identification RFID Technology and its Applications in the Commercial Construction Industry, Master Thesis, University of Kentucky

[4] Tu YJ, Zhou W and Piramuthu S (2009) Identifying RFID-embedded objects in pervasive healtcare applications, Decision Support Systems, vol. 46 , pp. 586-593

[5] Franke H and Dangelmaier W (2004) A web-based multi-agent system for transportation management to protect our natural environment, Cybernetics and Systems, vol. 35:7, pp. 627-638

[6] Liu ZG, Yue SH, Mei JQ and Zhang J (2009)Traffic video-based moving vehicle detection and tracking in the complex environment, $\mathrm{Cy}-$ bernetics and Systems, vol. 40: 7, pp. 569-588

[7] Böse F, Piotrowski J and Scholz-Reiter B (2008) Autonomously controlled storage management in vehicle logistics-applications of RFID and mobile computing systems, International Journal of RF Technologies: Research and Applications, pp. 1-20,

[8] Zoghi H, Tolouei M, Siamardi K and Araghi P (2008)Usage of ITS in the In-vehicle Signing System with RFID tags and Vehicle Routing and Road Traffic Simulation, 11th International IEEE Conference on Intelligent Trasportation Systems, pp. 408-413

[9] Jinghui Q, Bo S and Qidi Y (2006) Study on RFID Antenna for Railway Vehicle Identification, 6th International Conference on ITS Telecommunications, pp. 237-240

[10] Antikainen H, Colpaert A, Jaako N, Rusanen J, Bendas D, Myllyaho M, Oivo M, Kuvaja P, Similä J, Marjoniemi K, Laine K and Saari E (2004) Mobile Environmental Information Systems, Cybernetics and Systems, vol. 35: 7, pp. 737-751

[11] Sun Z. and (Jeff) Ban X. (2013) Vehicle classification using GPS data, Transportation Research Part C: Emerging Technologies, Volume 37 , pp. 102-117.

[12] El-Rabbany (2006) A Introduction to GPS: The Global Positioning System 2nd Ed., Artech House

[13] Psiaki, M. L.; O'Hanlon, B. W.; Bhatti, J. A.; Shepard, D. P.; Humphreys, T. E. (2013) GPS Spoofing Detection via Dual-Receiver Correlation of Military Signals, Aerospace and Electronic Systems, IEEE Transactions on, vol.49, no.4, pp.2250-2267.

[14] Park S.; Lee H. (2013) Self-Recognition of Vehicle Position Using UHF Passive RFID Tags, Industrial Electronics, IEEE Transactions on, vol.60, no.1, pp.226-234.

[15] Liu, S., Wang, X., Shen, J., Wang, B., Ye, T., \& Huang, R. (2014). A novel low-noise high-linearity CMOS transmitter for mobile UHF RFID reader. Science China Information Sciences, 1-8.

[16] Ward M and van Kranenburg R (2006) RFID:Frequency, Standards, Adoption and Innovation, JISC Technology and Standards Watch

[17] Kucukkomurler A (2009) Thermoelectric Powered High Temperature Wireless Sensing, Journal of Thermal Science and Technology, vol. 4, no. 1 , pp. 63-73

[18] Ragul, M., \& Venkatesh, V. (2013). Autonomous vehicle transportation using wireless technology. International Journal of Engineering and Technology (IJET), 5(2).

[19] Apaydin, S. F., Çavuşoğlu, A., \& Lami, K. A. Y. A. (2013). The Experiments And Performance Analyzes Of The Tracker Robots In Different Lighting Environments. International Journal, 2(1), 23051493. 